\title{
AI, Organizing, and Management
}

\author{
Aron Lindberg \\ Stevens Institute of Technology \\ aron.lindberg@stevens.edu
}

\author{
Jeffrey V. Nickerson \\ Stevens Institute of Technology \\ jnickers@stevens.edu \\ Stefan Seidel \\ University of Liechtenstein \\ stefan.seidel@uni.li
}

\author{
Jeffrey Saltz \\ Syracuse University \\ jsaltz@syr.edu
}

As organizations increasingly use artificial intelligence (AI) technologies to improve their operations [1] and as we see the emergence of novel forms of human-AI hybrids [2], we need new management theories, frameworks, and methodologies that can help us get at the relationship between AI, organizing, and management [3]. AI-based agents often rely on complex internal processing and their behavior is less predictable than that of the types of IT artifacts we are used to dealing with [4]. Consequently, any theorizing about the role of AI for organizing and management needs to attend to the material properties of AI-based systems [5].

We contend that information systems, computer science, and related fields have a two-pronged role in this context as both explanatory and predictive sciences and prescriptive sciences [6]. First, in the spirit of explanatory and predictive science, they can contribute to our understanding of how AI systems revamp organizational structures and require novel management strategies. Second, in the spirit of prescriptive science, both academic and industry researchers can design interventions that help organizations develop strategies intended to facilitate the management of AI or that are based on AI. Such strategies are likely to be based on prescriptive knowledge that spells out how to implement AI solutions in a way that considers organizational factors.

The two prongs of explanation/prediction and prescription are reflected in the papers that were submitted to this track, which cover a range of methodological perspectives, ranging from explanation to design.

Four papers will be presented, each addressing different aspects of AI, organizing, and management. The first paper, "Innovating with Artificial Intelligence: Capturing the Generative Functional Capabilities of Deep Generative Learning" [7], identifies a set of functional capabilities of Deep Generative Learn- ing (DGL) to generate outputs, and the authors discuss DGL's potential for innovation. The second paper, "Computer, Whom Should I Hire?-Acceptance Criteria for the Usage of Artificial Intelligence in the Recruitment Process" [8], identifies factors that influence AI acceptance in the context of the recruitment process. The third paper, "Opening the Black Box of Music Royalties with the Help of Hybrid Intelligence" [9], proposes design requirements for hybrid intelligence systems in the music industry. The authors use a design science research approach to present a prototypical instantiation. Finally, the fourth paper, "Can AI Become a State Servant? A Case Study of an Intelligent Chatbot Implementation in a Scandinavian Public Service" [10], describes and analyzes a chatbot in the customer center of a public service entity. The authors highlight how the implementation affected job characteristics such as skill variety, autonomy, and feedback.

These articles provide a taste of the varied implications of AI for organizing and managementaddressing algorithmic capabilities, techniques of designing AI systems for particular organizational contexts, and, more broadly, the ways that AI-based systems change the nature of work.

\section{References}

[1] M. Tarafdar, C. M. Beath, and J. W. Ross, "Using AI to Enhance Business Operations," MIT Sloan Management Review, vol. 60, no. 4, pp. 37-44, 2019.

[2] A. Rai, P. Constantinides, and S. Sarker, "Editor's Comments: Next-Generation Digital Platforms: Toward Human-AI Hybrids," Management Information Systems Quarterly, vol. 43, no. 1, pp. iii-ix, 2019. 
[3] N. Berente, B. Gu, J. Recker, and R. Santhanam, "Managing AI," Call for Papers, MIS Quarterly, 2019.

[4] B. Xiao and I. Benbasat, "E-Commerce Product Recommendation Agents: Use, Characteristcs, and Impact," MIS Quarterly, vol. 31, no. 1, pp. 137-209, 2007.

[5] S. Seidel, N. Berente, A. Lindberg, K. Lyytinen, B. Martinez, and J. V. Nickerson, "Artificial Intelligence and Video Game Creation: A Framework for the New Logic of Autonomous Design," Journal of Digital Social Research, vol. 2, no. 3, pp. 126-157, 2020.

[6] S. Seidel and R. Watson, "Integrating Explanatory/Predictive and Prescriptive Science in Information Systems Research," Communications of the Association for Information Systems, vol. 47, no. Paper 14, pp. 284-314, 2020.

[7] P. Hofman, T. Rückel, and N. Urbach, "Innovating with Artificial Intelligence: Capturing the Constructive Functional Capabilities of Deep Generative Learning," in 54th Hawaii International Conference on System Sciences (HICSS), 2021.

[8] V. Laurim, S. Arpaci, B. Prommegger, and H. Krcmar, "Computer, Whom Should I Hire? - Acceptance Criteria for Artificial Intelligence in the Recruitment Process," in 54th Hawaii International Conference on System Sciences (HICSS), 2021.

[9] E. Elshan, C. Engel, and P. Ebel, "Opening the Black Box of Music Royalties with the Help of Hybrid Intelligence," in 54th Hawaii International Conference on System Sciences (HICSS), 2021.

[10] A. Henk and F. Nilssen, "Can AI Become a State Servant? A Case Study of an Intelligent Chatbot Implementation in a Scandinavian Public Service," in 54th Hawaii International Conference on System Sciences (HICSS), 2021. 